phys. stat. sol. (b) 188, 43 (1995)

Subject classification: 71.35 and $78.66 ; 71.36 ; \mathrm{S} 7.12 ; \mathrm{S} 7.15$

Center for Ultrafast Optical Science, The University of Michigan, Ann Arbor ${ }^{1}$ )

\title{
Excitonic Radiative Dynamics in Multiple Quantum Wells
}

By

D. S. Citrin

The fundamental intrinsic radiative process associated with excitons in semiconductor multiple quantum wells is discussed within the context of exciton polaritons. Multiple quantum wells in which each well is randomly displaced from its ideal periodic position are discussed in order to investigate the effect of disorder on excitonic radiative widths. The coherent transport of excitons in multiple quantum wells is discussed.

\section{Introduction}

Although it is debatable who first introduced the concept of exciton polariton, it was Hopfield's famous paper of 1958 which set the tone for much of the later work on the topic [1]. The term exciton polariton was originally used by Hopfield to describe the stationary coupled modes involving an exciton and light in ideal infinite bulk crystals. Although the theoretical technique employed was to diagonalize the Hamiltonian for the crystal interacting with the quantized modes of the electromagnetic field, it was also realized that the exciton polaritons correspond to resonances in the dielectric function. Underlying this was an apparant dichotomy later to emerge, namely of exciton polaritons as viewed as resonances in some quantity characterizing the excitons (material approach) or as resonances in some quantity characterizing light propagation through the medium (opitcal approach). Whether exciton polaritons are viewed as elementary excitations or as resonances in the dielectric function, the same underlying physics is involved. Nevertheless, one approach or the other may be more convenient for the problem at hand. In the paper by Andreani [22], exciton polaritons in multiple quantum wells (MQW) are discussed in the context of the optical approach; in the present paper exciton polaritons are discussed employing the material approach.

At this point let us clarify what we mean by an exciton polariton. As it shall shortly be made clearer, the term exciton polariton here denotes a resonance in a correlation function (e.g. Green's function, GF) for a material degree of freedom (e.g. the spectrum of the exciton number operator) in the presence of the self-consistent interaction with the electromagnetic field. A corresponding definition applies in the time domain. The model Hamiltonian is to include only terms quadratic in exciton and photon creation and anihilation operators, and the exciton operators are assumed bosonic. In the first place, why concern ourselves with exciton polaritons? The answer is simply that they are the elementary excitations most closely associated with light emission by the radiative recombination of excitons. They provide a minimal model for light emission from quantum structures, in that we need not invoke the participation of other elementary excitations or

1) 2200 Bonisteel Boulevard, Ann Arbor, MI 48109-2099, USA. 
of static disorder - despite the major roles played by both - in order to discuss the fundamental radiative process. In addition, we shall see that polariton effects give rise to a novel mechanism for vertical exciton transport in MQWs. In short, the concept of the exciton polariton is key in order to describe the radiative dynamics of excitons on ultrafast time scales comparable or short compared with the dephasing processes due to other scattering mechanisms.

In this paper we discuss radiative dynamics involving excitons in MQWs in the context of the material approach to exciton polaritons. By way of introduction, we start with a brief discussion of exciton polaritons. A fundamental point is that polaritons involve a spatially coherent coupling of the exciton with the optical field over a length scale not much smaller than the wavelength in the medium of the emitted light. Consider the case when the coherence is complete in directions of translational invariance in a structure. In bulk, excitons are described (in an effective-mass treatment) by three-dimensional plane waves in the center-of-mass coordinate. Energy-momentum conservation dictates bounds on the momentum of those free excitons that can decay into a photon. For an infinite bulk crystal, only those states exactly at the crossing of the exciton and photon dispersions can decay (see Fig. 1a). The coupling between the exciton and the electromagnetic field, however, gives rise to an anticrossing in these dispersions. Hence, no coupled mode resides at the noninteracting crossing and consequently exciton polaritons in ideal infinite bulk crystals

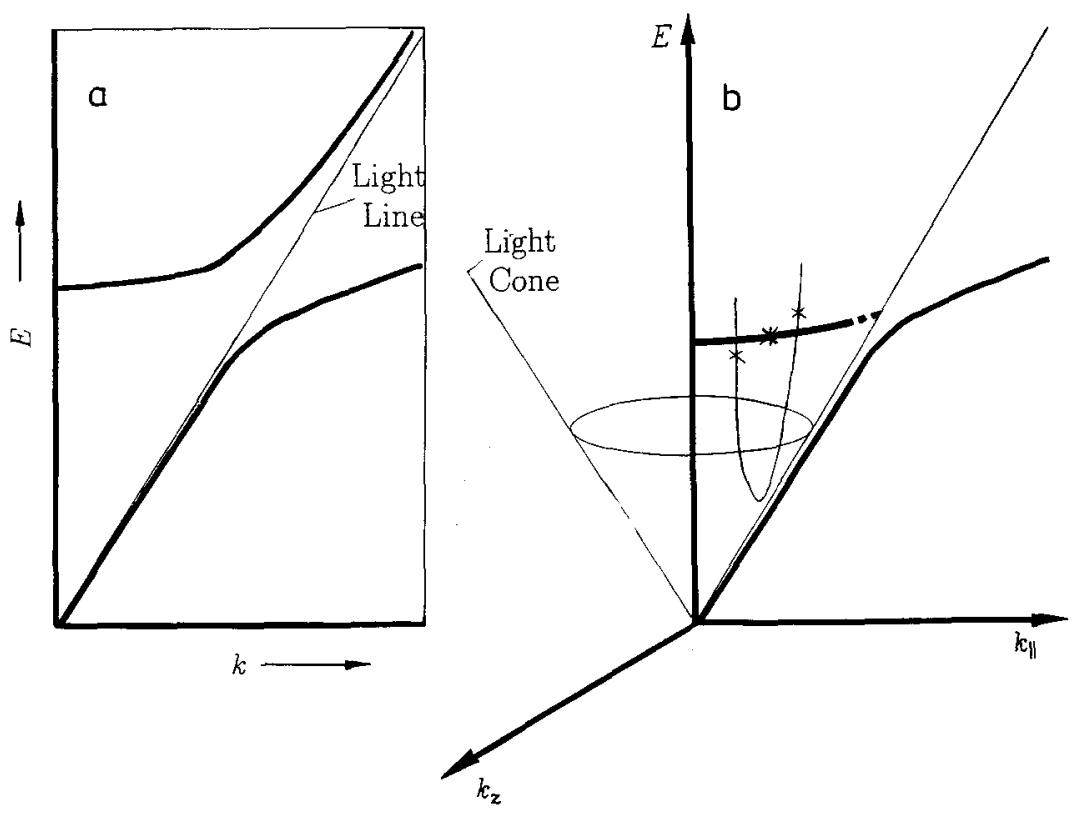

Fig. 1. Schematic exciton-polariton dispersion (heavy curves) for a) bulk and b) a QW. For bulk, all polariton states are stationary due to the absence of available electromagnetic modes to conserve energy and momentum in the decay process; only at the point where the two dispersions cross decay would be allowed. For the QW, the exciton dispersion is confined to the plane, but the photons are not. Thus, each exciton at $\boldsymbol{k}_{\|}$interacts with all the photons lying on the hyperbola with the same $\boldsymbol{k}_{\|}$. For states lying inside the light cone, i.e. with $k_{\|}<\chi$, photons with $k_{z} \neq 0$ can carry away the extra momentum necessary to conserve energy and momentum. The polariton state marked $*$ can decay into the photons marked $x$ 
are stationary with respect to the radiative decay process. In order to have irreversible decay, the point at the noninteracting crossing must somehow be accessed so that energymomentum conservation can be satisfied in the decay process. Scattering by phonons, defects, impurities, or crystal interfaces in practice accomplish this. The situation with lowdimensional semiconductors is entirely different. By way of illustration, consider a quantum well (QW). The absence of translational symmetry in the growth direction implies that the normal component of the quasi-momentum is no longer a good quantum number. Thus, a QW exciton with some given in-plane center-of-mass wave vector $\boldsymbol{k}_{\|}$interacts with a continuum of photons with the three-dimensional wave vector $\boldsymbol{k}=\left(\boldsymbol{k}_{\|}, \boldsymbol{k}_{z}\right)$ though having the same value of $\boldsymbol{k}_{\|}$(see Fig. 1 b). It is found that only the excitons lying inside the light cone may undergo a radiative decay [ 2 to 5] while energy-momentum conservation prohibits the decay of states with larger $\boldsymbol{k}_{\|}$. This is the lowest-order picture of radiative decay. In real structures, static disorder, phonons, exciton-exciton, and exciton-free-carrier scattering all play important parts depending upon sample quality, temperature, and excitation conditions. We shall concentrate on the lowest-order picture in Section 2. In Section 3 we touch upon several topics related to the role of static disorder. Section 4 considers the coherent vertical transport of excitons in MQWs. We give our concluding remarks in Sections 5 .

\section{Theory of Polaritons in Quantum Wells}

Before advancing to the topics of the ensuing sections, we here give a brief overview of exciton polaritons in single QWs and MQWs approached from the viewpoint of the material, i.e. excitonic, degrees of freedom, rather than from the alternative perspective of the external electromagnetic degrees of freedom. The method for carrying out our study is based on the GF for the exciton creation operator, the general techniques for which can be found for example in [7]. The GF can be related directly to basic experimental properties of the system, such as the exciton population dynamics and the electromagnetic spectrum of the spontaneously emitting excitons.

The central quantity is the self-energy due to the self-consistent interaction of the oscillating dipole moment associated with the exciton crystal ground state transition with the electromagnetic field. To save space, we consider an infinite, periodic MQW; the results will be specialized to the single QW below. The matrix elements between QWs $l$ and $m$ of the time-ordered (zero-temperature) GF for the exciton transition dipole moment are

$$
D_{l m}\left(t, \boldsymbol{k}_{\|}\right)=-i\left\langle 0\left|T b_{l \boldsymbol{k} \|}(t) b_{m \boldsymbol{k}_{\|}}^{\dagger}(0)\right| 0\right\rangle=-i\left\langle T b_{1 \boldsymbol{k}_{\|}}(t) b_{m \boldsymbol{k}_{\|}}^{\dagger}(0)\right\rangle,
$$

where $|0\rangle$ is the crystal ground state, $\langle T \ldots\rangle$ is the time-ordered zero-temperature expectation value, and $b_{l \boldsymbol{k}_{\|}}(t)$ is the Heisenberg-picture destruction operator for dipole excitation on QW $l$ with in-plane wave vector $\boldsymbol{k}_{\|}$. We shall write $\mathbf{D}$ to denote the matrix GF. Standard manipulations may be employed to obtain the GF as a function of frequency (energy) rather than time [7]. We can write the inverse GF as

$$
\mathbf{D}^{-1}=\mathbf{D}^{\prime-1}-\hbar \Sigma^{(1)} \mathbf{F},
$$

where $\hbar \Sigma^{(1)} \mathbf{F}$ is a self-energy (SE) matrix (to be discussed below) and

$$
\mathbf{D}^{\prime}=\frac{2 E_{\mathrm{ex}}\left(\boldsymbol{k}_{\|}\right) \mathbf{1}}{\zeta^{2}-E_{\mathrm{ex}}^{2}\left(\boldsymbol{k}_{\|}\right)-2 E_{\mathrm{ex}}\left(\boldsymbol{k}_{\|}\right) \hbar \Sigma^{(2)}} .
$$


$\hbar \Sigma^{(2)}$ is another SE term to be discussed shortly. For the infinite MQW the GF is diagonal in the normal-direction plane-wave basis $\{|q\rangle\}$ [6]

$$
D_{q}(k)=\frac{2 E_{\mathrm{ex}}\left(\boldsymbol{k}_{\|}\right)}{\zeta^{2}-E_{\mathrm{ex}}^{2}\left(\boldsymbol{k}_{\|}\right)-2 E_{\mathrm{ex}}\left(\boldsymbol{k}_{\|}\right)\left[\hbar \Sigma^{(2)}+\hbar \Sigma^{(1)}(k) F_{q}^{(0)}\right]},
$$

where $[\mathbf{D}]_{q q^{\prime}}=D_{q} \delta_{q q^{\prime}}, q$ is the normally directed $(z)$ component of the excitation wave vector, $\zeta$ is the complex energy variable, and $k=\left(\boldsymbol{k}_{\|}, \zeta\right) ; E_{\mathrm{ex}}\left(\boldsymbol{k}_{\|}\right)$is the $\boldsymbol{k}_{\|}$-dependent exciton energy, $\alpha=\left[k_{\|}^{2}-\varkappa^{2}\right]^{1 / 2}$, and $\chi=\zeta /(h c)$. $\alpha$ is the wave vector in the $z$-direction associated with the electromagnetic component of the polariton which mediates the interwell coupling. The superscript 0 on $F_{q}^{(0)}$ denotes the infinite periodic case, i.e., $F_{q q^{\prime}}=F_{q}^{(0)} \delta_{q q^{\prime}}$. For $k_{\|}<\varkappa$ $\in \mathscr{R}^{+}, \alpha$ is imaginary and the electromagnetic field is propagating; for $k_{\|}>x \in \mathscr{R}^{+}, \alpha$ is real and the associated field is evanescent. The speed of light in the dielectric medium in the absence of the exciton resonance of interest is $c=c_{0} / \sqrt{\varepsilon_{b}}$, where $c_{0}$ is the speed of light in vacuum and $\varepsilon_{\mathrm{b}}$ the background dielectric constant (assumed spatially uniform) which in practice we can take to be the high-frequency value. The GF for the single $Q W$ is obtained by setting $F_{q}^{(0)}=1$; thus the sum of $\hbar \Sigma^{(1)}$ and $\hbar \Sigma^{(2)}$ is the single-QW SE. For the MQW, F describes the interwell coupling.

The exciton-polariton modes in a symmetric single QW can be categorized by the symmetry of the associated electromagnetic field. We are concerned only with the dipole-active modes since the inactive mode does not contribute to either the radiative decay or the transport properties in the absence of scattering. The dipole-active modes are each associated with a direction $\hat{\boldsymbol{n}}_{\varepsilon}$ of the transition dipole moment:

$$
\begin{array}{ll}
\varepsilon=\mathrm{T} ; & \hat{n}_{\mathrm{T}} \cdot \boldsymbol{k}_{\|}=\hat{n}_{\mathrm{T}} \cdot \hat{z}=0, \\
\varepsilon=\mathrm{L}, & \hat{n}_{\mathrm{L}} \cdot \boldsymbol{k}_{\|}=\boldsymbol{k}_{\|}, \\
\varepsilon=\mathrm{Z} ; & \hat{n}_{\mathrm{Z}} \cdot \hat{z}=\hat{z} .
\end{array}
$$

The three kinds of polaritons are denoted $T, L$, and $Z$. For modes possessing mirror symmetry in the $z$-direction, the modes $\mathrm{L}$ and $\mathrm{Z}$ are uncoupled. We shall in general neglect the $\mathrm{L}-\mathrm{Z}$ coupling. (See, however, the paper of Andreani [22]).

The explicit form of $\hbar \Sigma_{\varepsilon}^{(1)}$ is $[5,6]$

$$
\hbar \Sigma_{\varepsilon}^{(1)}(\boldsymbol{k})= \begin{cases}-\frac{2 \pi}{\alpha} \frac{\zeta^{2}}{E_{\mathrm{ex}}\left(\boldsymbol{k}_{\|}\right)^{2}}\left|C_{s_{\mathrm{T}}} \cdot \hat{n}_{\mathrm{T}}\right|^{2}|S|^{2} ; & \text { T-mode, } \\ \frac{2 \pi}{\alpha} \frac{\zeta^{2}}{E_{\mathrm{cx}}\left(\boldsymbol{k}_{\| 1}\right)^{2}} \frac{\alpha^{2}}{\chi^{2}}\left|C_{s_{\mathrm{L}}} \cdot \hat{n}_{\mathrm{L}}\right|^{2}|S|^{2} ; & \text { L-mode, } \\ -\frac{2 \pi}{\alpha} \frac{\zeta^{2}}{E_{\mathrm{ex}}\left(\boldsymbol{k}_{\|}\right)^{2}} \frac{k_{\|}^{2}}{\varkappa^{2}}\left|C_{s_{\mathrm{Z}}} \cdot \hat{\boldsymbol{n}}_{\mathrm{Z}}\right|^{2}|S|^{2} ; & \text { Z-mode } .\end{cases}
$$

Here $S=\int \mathrm{d} z\left|f_{\mathrm{c}}(z) f_{\mathrm{v}}(z)\right|$, where $f_{\mathrm{v}}(z)\left[f_{\mathrm{c}}(z)\right]$ is the valence- (conduction-) band envelope function, and $C_{s}$ the coupling strength. $C_{s}$ is proportional to the transition dipole moment, $C_{s}=\varkappa_{\mathrm{ex}} \varepsilon_{\mathrm{b}}^{-1 / 2} U_{\mathrm{ex}}(\mathbf{0})\left\langle(c v)_{\mathrm{s}}|e \boldsymbol{R}| 0\right\rangle$, where $\varkappa_{\mathrm{ex}}=E_{\mathrm{ex}}\left(\boldsymbol{k}_{\| !}\right) /(\hbar c), U_{\mathrm{ex}}\left(\boldsymbol{r}_{\|}\right)$is the exciton envelope function for the electron-hole relative motion, $\left|(c v)_{s}\right\rangle$ is the electron-hole pair of spin $s$, and $\left\langle(c v)_{s}|e \boldsymbol{R}| 0\right\rangle$ is the dipole matrix element between $\left|(c v)_{s}\right\rangle$ and $|0\rangle . \boldsymbol{C}_{s}$ is related to the areal oscillator strength $f_{\varepsilon}$ by $f_{\varepsilon}=\left.2 m_{0} E_{\text {ex }}(\mathbf{0})\left|U_{\text {ex }}(\mathbf{0})\right|^{2} \hbar^{-2} \sum_{s}\left|\langle c v)_{s}\right| \boldsymbol{R} \cdot \hat{\boldsymbol{n}}_{\varepsilon}|0\rangle\right|^{2}$ with $m_{0}$ the 
free-electron mass. Where $\hbar \Sigma^{(1)}$ is well behaved, $-2 \operatorname{Im} \Sigma^{(1)}$ corresponds to the excitonic radiative decay rate as calculated from Fermi's Golden Rule [4].

$\hbar E_{\varepsilon}^{(2)}$ gives to the (LT)-Z splitting [8]. Its reads

$$
\hbar \Sigma_{\varepsilon}^{(2)}\left(\boldsymbol{k}_{\|}\right)=\frac{4 \pi}{\chi_{\mathrm{ex}}^{2}} \delta_{\varepsilon Z}\left|C_{s_{Z}} \cdot \hat{\boldsymbol{n}}_{\mathrm{Z}}\right|^{2} \mathscr{I}
$$

where $\mathscr{I}=\int \mathrm{d} z\left|f_{\mathrm{c}}(z) f_{\mathrm{v}}(z)\right|^{2}$. We thus have $\hbar \Sigma^{(2)}=0$ unless $\varepsilon=\mathrm{Z}$, in which case $\hbar \Sigma^{(2)}$ is a real constant independent of $(q, k) . h \Sigma^{(2)}$ therefore plays no significant dynamical role.

$\mathbf{F}$ describes the interwell coupling. The direct-space matrix elements of $\mathbf{F}$ are

$$
F_{l m}=\mathrm{e}^{-\alpha\left|L_{l}-L_{m}\right|},
$$

where $L_{l}$ is the position of the $l$-th QW. For the infinite periodic MQW, $\mathbf{F}$ is diagonal in the plane-wave basis,

$$
F_{\mathrm{q}}^{(0)}=\frac{\sinh y}{\cosh y-\cos \varphi}=\frac{\sinh y}{2\left(\sinh ^{2} \frac{y}{2}+\sin ^{2} \frac{\varphi}{2}\right)}
$$

with $y=\alpha L$ and $\varphi=q L$. Here $L$ is the periodicity of the MQW in the $z$-direction. Thus, for $k_{\|}>|x|(\varkappa<\mathscr{R}), \alpha$ is real and the coupling strength falls off exponentially. For $k_{\|}<|x|$, however, the relevant values of $\alpha$ are imaginary, and consequently the coupling is long-ranged. It is easily verified that $\hbar \Sigma^{(1)} F_{q}^{(0)}$ is real. Consequently, there is no excitonic radiative decay in the infinite periodic MQW. This is expected, based upon what we know about bulk polaritons [1].

\section{Disorder Effects}

Static disorder of various kinds is always present, even in the best of QWs. In single QWs fabricated out of direct-gap materials disorder such as that due to interface roughness mixes states with different $\boldsymbol{k}_{\|}$. Consequently, the low-lying states are not pure $\boldsymbol{k}_{\|}=\mathbf{0}$, but some admixture with a spread in $\boldsymbol{k}$-space given by the inverse of the localization length [5]. This leads to relatively long radiative recombination times for the low-lying excitons [5], which shows up as slow decay of the time-resolved photoluminescence signal at low temperature [9]. For a review of these effects, see [10]. In QWs formed from indirect-gap materials possessing type-I excitons, disorder of this sort is expected to enhance the low-temperature emission rate [11]. In this section we consider a different type of disorder. Namely, we consider an infinite MQW composed of identical QWs in which each QW is displaced slightly by a random amount from its periodic positions. This model is of interest because it provides a minimal model for an infinite system for the study of the disorderinduced origin of the excitonic radiative decay.

Let us specify our model. The MQW stack is composed of $N$ identical symmetric QWs ( $N$ shall be taken to infinity) each of width $L_{z} . L_{z}$ is assumed to be much less than the wavelength of the emitted light in the medium. The structure is assumed to posses strict in-plane translational invariance. In the growth direction, however, each QW is slightly shifted from the position it would occupy in a periodic structure. Moreover, we assume that the disorder is uncorrelated between distinct QWs $l$ and $l^{\prime}$. The almost-periodic character of the MQW suggests that a convenient starting point for a theoretical description of the 
polaritons is provided by a plane-wave basis $\{|q\rangle\}$ in the $z$-direction, where $\langle z \mid q\rangle$ $=N^{-1 / 2} \exp \{i q L l\}$ with $q \in\{2 \pi n / N L, n=0,1, \ldots, N-1\}$. Because this basis is orthonormal and $\mathbf{D}^{\prime}$ is diagonal in $\{|l\rangle\}, \mathbf{D}^{\prime}$ remains diagonal in the representation $\{|q\rangle\}$. The matrix $\mathbf{F}$, however, couples distinct states $|q\rangle$ and $\left|q^{\prime}\right\rangle$ :

$$
\begin{aligned}
F_{q q^{\prime}} & =\lim _{N \rightarrow \infty} \frac{1}{N} \sum_{l, l^{\prime}=1}^{N} \mathrm{e}^{i q L l} F_{l l^{\prime}} \mathrm{e}^{-i q^{\prime} L l^{\prime}} \\
& =\lim _{N \rightarrow \infty} \frac{1}{2 \pi} \int_{-\infty}^{\infty} \mathrm{d} p \frac{2 \alpha}{\alpha^{2}+p^{2}} \mathscr{S}(p, q) \mathscr{S}^{*}\left(p, q^{\prime}\right) .
\end{aligned}
$$

Here $\mathscr{S}(p, q)=N^{-1 / 2} \sum_{l=1}^{N} \exp \left\{i q L_{l}\right\} \exp \{i p L l\}$. In order to obtain the spectral and dynamical properties of the system, we must invert $\mathbf{D}^{-1}$ - an infinite matrix. As discussed in the previous section, for the periodic case $L_{l}=l L$, the basis $\{|q\rangle\}$ diagonalizes the problem [6]. The addition of the disorder via $L_{l}=l L+\chi_{l}$ with $\left|\chi_{l}\right|<L$ will restore, at least in part, the radiative width present in single QWs [12] but absent in the infinite periodic MQW.

The introduction of disorder through $\chi_{l} \neq 0$ leads states with different $q$ to become coupled giving rise again to a problem of infinite dimension. In order to make headway, we calculate the configuration average of the GF rather than the GF itself [13]. In the case when the disorder is instead in the site-diagonal part of the GF, the lowest-order term in the configuration average leads to the so-called virtual-crystal approximation (VCA). The VCA effectively results in a fictitious uniform medium with parameters that are in some sense the average of those characterizing the individual constituents of the mixed crystal. We shall find for our off-diagonal disorder model that the lowest-order approximation to the configuration average gives the leading modification to the radiative dynamics due to the random interwell spacings.

Let the configuration average of the quantity $\mathcal{O}$ be denoted $\langle\mathcal{O}\rangle$. Upon inverting $\mathbf{D}$ and performing the configuration average, one finds terms containing factors of the form $\left\langle\mathscr{S}\left(p_{1}, q_{1}\right) \mathscr{S}^{*}\left(p_{1}, q_{1}^{\prime}\right) \ldots \mathscr{S}\left(p_{m}, q_{m}\right) \mathscr{S}^{*}\left(p_{m}, q_{m}^{\prime}\right)\right\rangle$. The expression above would then factor as $\left\langle\mathscr{S}\left(p_{1}, q_{1}\right)\right\rangle\left\langle\mathscr{S}^{*}\left(p_{1}, q_{1}^{\prime}\right)\right\rangle \ldots\left\langle\mathscr{S}\left(p_{m}, q_{m}\right)\right\rangle\left\langle\left\langle\mathscr{S}^{*}\left(p_{m}, q_{m}^{\prime}\right)\right\rangle\right.$ except for the occurrence of terms which involve multiple occupancy on the same site. These correlations occur between any pair of $\mathscr{S}$ in the product; the dominant contributions come, however, from products of $\mathscr{S}$ with the same $p$-variable because other pairings are, on average, weak due to their essentially random phases. Thus, we replace the configuration average of the product with the factorization $\left.\left\langle\mathscr{S}\left(p_{1}, q_{1}\right) \mathscr{S}^{*}\left(p_{1}, q_{1}^{\prime}\right)\right\rangle \ldots \mathscr{S}\left(p_{m}, q_{m}\right) \mathscr{S}^{*}\left(p_{m}, q_{m}^{\prime}\right)\right\rangle$. We can now write down a Dyson equation for $\langle\mathbf{D}\rangle$ within these approximations as

$$
[\langle\mathbf{D}\rangle]^{-1} \approx\left\langle\mathbf{D}^{-1}\right\rangle=\mathbf{D}^{-1}-\left\langle\hbar \boldsymbol{\Sigma}^{(1)}\right\rangle,
$$

where $\hbar \Sigma^{(1)}=\hbar \Sigma^{(0)} \mathbf{F}$. We must therefore calculate $\left[\left\langle\hbar \Sigma^{(1)}\right\rangle\right]_{q q^{\prime}}$, which is proportional to $\left\langle\mathscr{S}(p, q) \mathscr{S}^{*}\left(p, q^{\prime}\right)\right\rangle$ :

$$
\left\langle\mathscr{P}(p, q) \mathscr{S}^{*}\left(p, q^{\prime}\right)\right\rangle=\frac{1}{N} \sum_{l, l^{\prime}=1}^{N} \mathrm{e}^{i(p+q) l L} \mathrm{e}^{-i(p+q) l^{\prime} L}\left\langle\mathrm{e}^{i p x x_{l}} \mathrm{e}^{-i p x^{\prime}}\right\rangle .
$$

Put $C=\left\langle\mathrm{e}^{i p \chi_{l}} e^{-i p \chi_{1^{\prime}}}\right\rangle$ and let the distribution $P(\chi)$ characterizing the disorder be defined such that $\left\langle\mathcal{O}\left(\chi_{l}\right)\right\rangle=\int_{-\infty}^{\infty} \mathrm{d} \chi P(\chi) \mathcal{O}(\chi)$. Furthermore, assume that $P(\chi)$ is normalized in the 
sense $\int_{-\infty}^{\infty} \mathrm{d} \chi P(\chi)=1$. If $\tilde{P}(p)=\int_{-\infty}^{\infty} \mathrm{d} \chi P(\chi) \mathrm{e}^{i p \chi}$, then $C=\left[1-|\tilde{P}(p)|^{2}\right] \delta_{l l^{\prime}}+|\tilde{P}(p)|^{2}$. This gives

$$
\left\langle\mathscr{P}(p, q) \mathscr{S}^{*}\left(p, q^{\prime}\right)\right\rangle=\sum_{G}\left\{\left[1-|\tilde{P}(p)|^{2}\right]+|\widetilde{P}(p)|^{2} N \sum_{G^{\prime}} \delta_{q, p+G^{\prime}}\right\} \delta_{q, q^{\prime}+G},
$$

where $G$ and $G^{\prime}$ are $\hat{z}$-direction reciprocal lattice vectors. To simplify what follows, we assume that $x_{\mathrm{ex}} L \ll 1$ holds. This allows us to neglect umklapp processes in the evaluation of integrals, namely we take $G, G^{\prime}=0$. We then have

$$
\begin{aligned}
& \left\langle F_{q q^{\prime}}\right\rangle=F_{q q^{\prime}}^{(0)}+\delta\left\langle F_{q q^{\prime}}\right\rangle, \\
& F_{q q^{\prime}}^{(0)}=F_{q}^{(0)}|\tilde{P}(q)|^{2} \delta_{q q^{\prime}}, \\
& \delta\left\langle F_{q q^{\prime}}\right\rangle=\frac{1}{2 \pi} \int_{-\infty}^{\infty} \mathrm{d} p \frac{2 \alpha}{\sigma^{2}+p^{2}}\left[1-|\widetilde{P}(p)|^{2}\right] \delta_{q q^{\prime}} .
\end{aligned}
$$

$F_{q}^{(0)}$ is given in (12); however, within our approximation of neglecting umklapp processes, we have $F_{q}^{(0)}=2 y /\left(y^{2}+\varphi^{2}\right)$. Thus, the approximation to the configuration-averaged $\mathrm{GF}$ $\left\langle\mathbf{D}_{\varepsilon}\right\rangle$ is also diagonal in the plane-wave representation $\{|q\rangle\}$, as it is in the ideal periodic case. The inversion of the inverse of the configuration-averaged GF is now simple

$$
[\langle\mathbf{D}\rangle]_{q q^{\prime}}=\frac{\delta_{q q^{\prime}}}{\mathbf{D}_{q q}^{\prime-1}-\left\langle\hbar \Sigma^{(1)}\right\rangle_{q q}} .
$$

With a choice for $P(\chi)$, we can calculate the disorder-induced broadening $-\operatorname{Im} \hbar \Sigma^{(1)}$. We can invoke the exciton-pole approximation [14], in which the energy $\zeta$ in the SE is replaced by $E_{\text {ex }}\left(\boldsymbol{k}_{\|}\right)$. If $\mid \operatorname{Im} \hbar \Sigma_{\varepsilon, q q}^{(1)}\left[E_{\text {ex }}\left(\boldsymbol{k}_{\|}\right)+i 0^{+}, \boldsymbol{k}_{\|]}\right] \ll E_{\text {ex }}\left(\boldsymbol{k}_{\|}\right)$, then $\hbar \Gamma_{\varepsilon}\left(\boldsymbol{k}_{\|}, q\right)=-\operatorname{Im} \hbar \Sigma_{\varepsilon, q q}^{(1)}\left[E_{\text {ex }}\left(\boldsymbol{k}_{\|}\right)\right.$ $\left.+i 0^{+}, \boldsymbol{k}_{\|}\right]$is the width of the spectral density at fixed $\left(\varepsilon, \boldsymbol{k}_{\|}, q\right)$. Below, we shall argue moreover that this quantity coincides with the radiative width of the state $\left(\varepsilon, \boldsymbol{k}_{\|}, q\right)$. Note that energy-momentum conservation dictates that the radiative width must vanish for $\hbar c k_{\|}>E_{\text {ex }}\left(\boldsymbol{k}_{\|}\right)$[3], which indeed $\hbar \Gamma_{\varepsilon}\left(\boldsymbol{k}_{\|}, q\right)$ does. $F_{q q^{\prime}}^{(0)}$ possesses a simple pole, and thus does not contribute directly to the radiative decay. We then have

$$
\Gamma_{\varepsilon}\left(\boldsymbol{k}_{\|}, q\right)=\Gamma_{\varepsilon}^{\mathrm{SQW}}\left(\boldsymbol{k}_{\|}\right) \operatorname{Re} \delta\left\langle F_{q q}\right\rangle,
$$

where $\Gamma_{\varepsilon}^{\mathrm{SQw}}\left(\boldsymbol{k}_{\|}\right)=-\operatorname{Im} \Sigma_{\varepsilon}^{(1)}\left[E_{\mathrm{ex}}\left(\boldsymbol{k}_{\|}\right)+i 0^{+}, \boldsymbol{k}_{\|}\right]$is the single-QW decay rate [5], which vanishes for $k_{\|}>E_{\mathrm{ex}}\left(\boldsymbol{k}_{\|}\right)$. In particular,

$$
\operatorname{Re} \delta\left\langle F_{q q^{\prime}}\right\rangle=\left\{1-\frac{1}{2}\left[|\widetilde{P}(\eta)|^{2}+|\tilde{P}(-\eta)|^{2}\right]\right\} \delta_{q q^{\prime}},
$$

where $\eta=\sqrt{x_{\mathrm{ex}}^{2}}-k_{\|}^{2}$. If the scale of variations in $P(\chi)$ is $d \geqq 0$, in the long-wavelength limit ( $\eta d \ll 1$ ), then $\operatorname{Re} \delta\left\langle F_{q q}\right\rangle \sim \eta^{2} d^{2}$, and so $\Gamma_{\varepsilon}\left(\boldsymbol{k}_{\|}, q\right) \approx \eta^{2} d^{2} \Gamma_{\varepsilon}^{\mathrm{SQw}}\left(\boldsymbol{k}_{\|}\right)$. ( $q d$ was already assumed small when we neglected umklapp processes). This can be seen explicitly with specific forms for the distribution $P$ [15].

To be certain, if we wish to compute the radiative width, we should calculate the time-dependent expectation value of the exciton number operator giving some initial one-exciton state. This quantity can be written as the sum of two-particle GF, each term 
of which is a product of two one-particle GF. For the MQW with disorder, we would then calculate the configuration average of the relevant two-particle GF. The decay of the time-dependence of the configuration-averaged number operator would then be attributable to radiative decay. The reason that the configuration-averaged one-particle GF is frequently inadequate to obtain the radiative width is that the disorder conceivably introduces density-of-states broadening without radiative broadening. For the problem at hand, however, we conjecture that the broadening of the configuration-averaged one-particle GF gives the radiative width since to the order we calculate the configuration average, we have $\langle\mathbf{D D}\rangle=\langle\mathbf{D}\rangle\langle\mathbf{D}\rangle$ for the configuration-averaged two-particle GF which we denote schematically as $\langle\mathbf{D D}\rangle$. Note, moreover, that the interaction does not result in broadening to lowest order for the excitons with $\hbar c k_{\|}>E_{\mathrm{cx}}\left(\boldsymbol{k}_{\|}\right)$.

\section{Coherent Vertical Exciton Transport}

In superlattices in which the barriers are sufficiently narrow and low, the low-temperature vertical transport of excitons is due to quantum-mechanical tunneling between successive quantum wells (QW) [16]. For structures with wide and high barriers, i.e. MQWs, the interwell coupling is due to the dynamic dipole-dipole interaction [17]. However, this is simply polariton effects in the time domain. Exciton transport has been of interest in various molecular systems (see for example [18]), where the picture is of incoherent Förster transport [19]. The MQW provides a tailor-made system of which an individual structure can be studied, while data on molecular systems must be frequently interpreted in terms of ensembles. There has been little experimental or theoretical [20] work on this type of vertical transport in MQWs, although incoherent transport in such structures has been the subject of one study [21].

In this section we study the coherent vertical transport of excitons in MQWs. An interesting result is that in planar systems there is a possibility of rapid coherent excitation transfer between different QWs. We consider the dynamics subsequent to the introduction of an initial exciton population into a single QW within the MQW. Schemes for doing so by means of incoherent excitation are dicussed. The envisaged experiment involves initial conditions markedly different from light propagation studies. The dynamics are governed by the coherent radiative decay (spontaneous emission) of excitons and reabsorption elsewhere in the structure. At low-temperature, where the occupied exciton states have very small in-plane momentum (direct-gap case), it is essential to consider the effects of retardation, i.e., the full dynamical diple-dipole interaction must be taken into account, since for small in-plane wave vectors $\boldsymbol{k}_{\|}$the interwell coupling including retardation is long-ranged, whereas the neglect of retardation arbitrarily introduces a finite spatial range to the interwell coupling. We consider an infinite, periodic MQW composed of symmetric, identical QWs, and assume that $L_{z}$ is much less than $\varkappa_{\mathrm{ex}}^{-1}$. The GF for the exciton transition dipole moment in QW $n$ following excitation with in-plane wave vector $k_{\|}$in QW 0 is $D_{n}\left(\mathrm{t}, \boldsymbol{k}_{\|}\right)$. The population in QW $n$ is then $N_{n}(t)=\left|D_{n}\left(t, \boldsymbol{k}_{\|}\right)\right|^{2}$. As we saw above, the GF is diagonal in the basis $\{|q\rangle\}$, and the dynamic dipole-dipole coupling, both intra and interwell, is characterized by a self-energy $\hbar \Sigma^{(1)} F_{q}^{(0)}$.

Recall that for $k_{\|}>\varkappa, \alpha$ is real indicating that the dynamic dipole-dipole interaction between distinct QWs is mediated by an evanescent electromagnetic field and the coupling strength falls off exponentially. For $k_{\|}<|x|$, however, the relevant values of $\alpha$ are imaginary (propagating field), and the coupling is long-ranged. The coupling energy $\hbar \Sigma^{(1)} F_{l m}$ is thus 
of a very different form from the Förster-type coupling between point dipoles $\left(r^{-3}\right)$. We now consider two limiting cases: (i) $k_{\|}>x, y \gg 1$. This reduces to the case of nearestneighbor dipole-dipole coupling between QWs (instantaneous limit, IL). (ii) $y \ll 1$ (long-wavelength approximation, LWA).

(i) For $k_{\|} \gg|\chi|$, retardation is negligible. The IL corresponds to an interwell interaction mediated by virtual photons throught the evanescent electric fields associated with large-wave vector polaritons. For $k_{\|} \gg|x|, \hbar \Sigma^{(1)}, y \in \mathscr{R}$. Since $y \gg 1$ by assumption, $\sinh y$, $\cosh y \gg 1$ and $F_{q}^{(0)} \approx \tanh y[1+\operatorname{sech} y \cos \varphi]$. In the pole and rotating-wave approximations, the Fourier time transform of $D_{q}(k)$ is

$$
\begin{aligned}
i D_{q}\left(t, \boldsymbol{k}_{\|}\right)= & \theta(t) \exp \left(-i\left(\tilde{E}+\tanh y \hbar \Sigma^{(1)}+G_{A} \cos \varphi\right) t / \hbar\right) \\
& +\theta(-t) \exp \left(i\left(\tilde{E}+\tanh y \hbar \Sigma^{(1)}+G_{A} \cos \varphi\right) t / \hbar\right)
\end{aligned}
$$

with $G_{A}=\tanh y \operatorname{sech} y \hbar \Sigma^{(1)}, \theta(t)=1$ if $t<0$ or 0 if $t>0$, and $\tilde{E}$ is a constant such as $E_{\text {ex }}\left(\boldsymbol{k}_{\|}\right)$. The spatial Fourier transform gives

$$
\begin{aligned}
i D_{n}\left(t, \boldsymbol{k}_{\|}\right)= & i^{n} \mathrm{~J}_{n}\left(\frac{G_{A} t}{\hbar}\right)\left[\theta(t) \exp \left(-i\left(\tilde{E}+\tanh y \hbar \Sigma^{(1)}\right) t / \hbar\right)\right. \\
& \left.+\theta(-t) \exp \left(i\left(\tilde{E}+\tanh y \hbar \Sigma^{(1)}\right) t / \hbar\right)\right],
\end{aligned}
$$

where $\mathrm{J}_{n}(x)$ is the Bessel function of first kind. Thus, the dynamics depend solely on $n$ and the scaled time $\tau_{A}=G_{\mathrm{A}} t / \hbar$. The population in QWs $n$ as a function of scaled time $N_{n}\left(\tau_{\mathrm{A}}\right)=\left|D_{n}\left(\tau_{\mathrm{A}}\right)\right|^{2}$ is plotted in Fig. $2 \mathrm{a}$. One finds for $\tau_{\mathrm{A}} \ll 1$,

$$
N_{n}(t) \approx \begin{cases}1-\frac{1}{2}\left(G_{\mathrm{A}} t / \hbar\right)^{2} ; & n=0, \\ \frac{1}{4} \frac{\left(G_{\mathrm{A}} t / \hbar\right)^{2|n|}}{(|n| !)^{2}} ; & n \neq 0 .\end{cases}
$$

As $t \rightarrow 0$, the time derivative of $N_{n}(t) \rightarrow 0$. This leads to a time delay for the transfer of excitation to QWs $n$ as seen in Fig. 2 a. For $\tau_{A} \gg|n|$, we obtain the long-time behavior

$$
N_{n}(t) \approx\left(\frac{2 \hbar}{\pi G_{A} t}\right) \cos ^{2}\left(\frac{G_{\mathrm{A}} t}{\hbar}-\frac{\pi|n|}{2}-\frac{\pi}{4}\right) .
$$

Thus, for large $\tau_{\mathrm{A}}$ the time scale for the loss of population in a given $\mathrm{QW}$ is $\hbar / G_{\mathrm{A}}$.

(ii) The LWA is valid at all $\boldsymbol{k}_{\|}$if $k_{\|} L \ll 1$. We have $F_{q} \approx y /(1-\cos \varphi)$. In the pole and rotating-wave approximations as in (i), we obtain

$$
i D_{n}\left(t, \boldsymbol{k}_{\|}\right)=\theta(t) \mathrm{e}^{-i \tilde{E} t / \hbar} R_{n}\left(\tau_{\mathbf{B}}\right)+\theta(-t) \mathrm{e}^{i \tilde{E} \tau / \hbar} R_{n}^{*}\left(\tau_{\mathbf{B}}\right),
$$

where

$$
\begin{aligned}
R_{n}\left(\tau_{\mathrm{B}}\right) & =\frac{1}{2 \pi} \int_{-\pi}^{\pi} \mathrm{d} \varphi \mathrm{e}^{-i \tau_{\mathrm{B}}(1-\cos \varphi)^{-1}} \mathrm{e}^{i n \varphi} \\
& =\frac{1}{2 \pi} \int_{-\pi}^{\pi} \mathrm{d} \varphi \mathrm{e}^{-i \frac{\tau_{\mathrm{B}}}{2} \csc ^{2} \frac{\varphi}{2}} \mathrm{e}^{i n \varphi}
\end{aligned}
$$



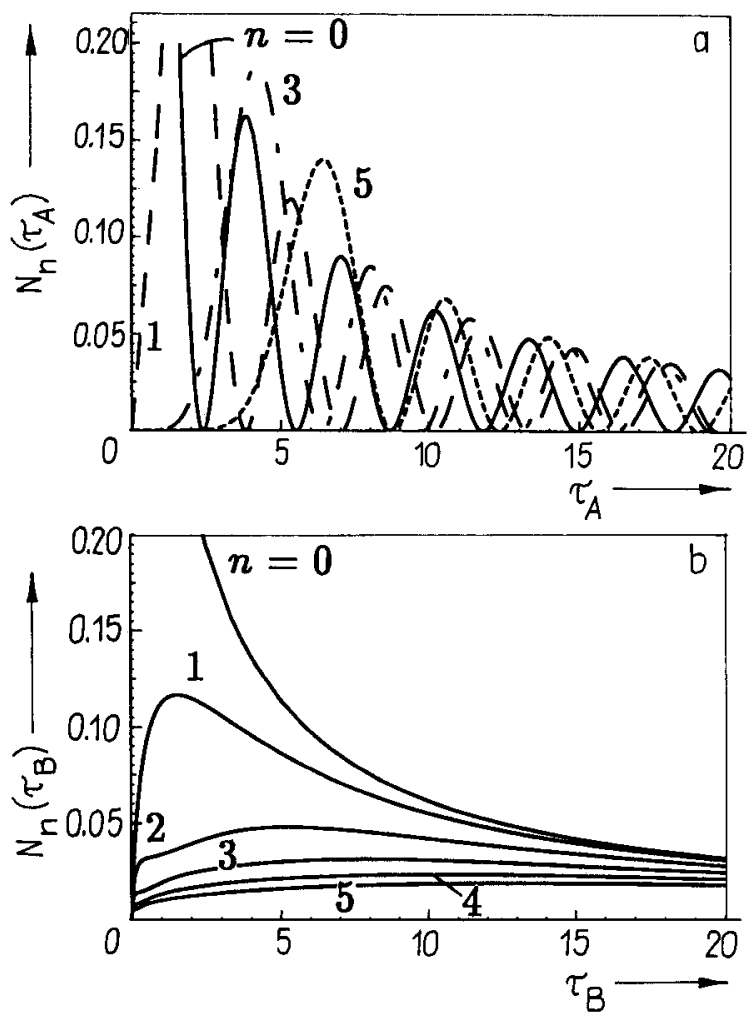

Fig. 2. The exciton population in QW $n$ as a function of scaled time. a) IL, b) LWA

with $\tau_{\mathbf{B}}=G_{\mathbf{B}} t / \hbar$ and $G_{\mathbf{B}}=\hbar \Sigma^{(1)} y$. We therefore see that in the LWA the population dynamics $N_{n}(t)=\left|R_{n}\left(\tau_{\mathrm{B}}\right)\right|^{2}$ depend only upon the function $R_{n}\left(\tau_{B}\right)$, indexed by $n$, of the single parameter $\tau_{\mathrm{B}}$, which describes the time scaled by $G_{\mathrm{B}} / \hbar$. The integral $R_{n}\left(\tau_{\mathrm{B}}\right)$ can be expressed in closed form, i.e., in terms of a finite number of standard special functions. Although the resulting expression is cumbersome, it is nevertheless useful in order to extract limiting behavior. It is convenient to define $\beta=i \tau_{\mathrm{B}} / 2$ and $z=\cos ^{2}(\varphi / 2)$. By making the change of the variable $\varphi \rightarrow z$, we obtain

$$
R_{n}=\frac{(-1)^{n}}{\pi} \sum_{p=0}^{|n|}(-1)^{p}{ }_{2|n|} C_{2 p} \int_{1}^{\infty} \mathrm{d} z z^{-|n|-1}(z-1)^{p-\frac{1}{2}} \mathrm{e}^{-\beta z},
$$

where ${ }_{2|n|} C_{2 p}$ is a binomial coefficient. The integral is then evaluated to give

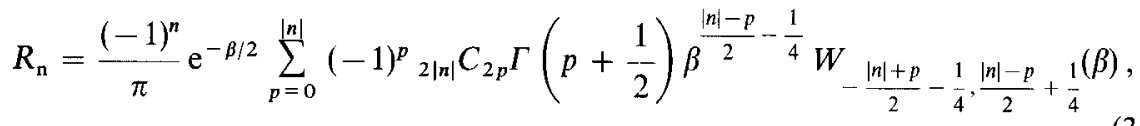

where $W_{\lambda, \mu}(\beta)$ is the Whittaker function. In particular we have at $n=0$

$$
R_{0}=\operatorname{erfc}\left(\beta^{1 / 2}\right)
$$

where erfe is the complementary error function. 
$N_{n}\left(\tau_{\mathrm{B}}\right)=\left|D_{n}\left(\tau_{\mathrm{B}}\right)\right|^{2}$ is shown in Fig. 2 b. In the short-time limit $\tau_{\mathrm{B}} \ll 1$, we obtain

$$
N_{n}(t)= \begin{cases}1-\sqrt{\frac{G_{\mathrm{B}} t}{\pi \hbar}} ; & n=0, \\ \frac{2 G_{\mathrm{B}} t}{\pi \hbar} ; & n \neq 0 .\end{cases}
$$

The time derivative of the population at $t=0$ does not vanish (for $n=0$ it diverges), unlike for the IL. For $n \neq 0$, the initial rise is independent of $n$. Thus, the long-range character of the coupling between QWs gives rise to fast excitation transfer, in contrast to what was seen in the IL. The long-time limit $\tau_{\mathbf{B}} \gg|n|$ gives

$$
N_{n}(t) \approx \frac{2 \hbar}{\pi G_{\mathrm{B}} t}
$$

Apart from the oscillatory factor in (i), the long-time dynamics are thus formally similar to what was found above in the IL. Because the LWA is a continuum limit, we do not expect the oscillatory part which arises from the discreteness of the MQW.

We summarize: (i) The IL corresponds to the neglet of retardation. In the extreme limit, we have $G_{\mathrm{A}} \sim \mathrm{e}^{-y} \hbar \Sigma^{(1)}$ and the dominant coupling is between nearest-neighbor QWs $n= \pm 1$, and the expressions for the GF are well known. The IL involves large-wave vector excitations which cannot couple directly with the free-space optical field. Time-domain techniques related to attenuated total internal reflection spectroscopy and near-field optical microscopy show promise to access these modes. For GaAs/AlGaAs-based structures,

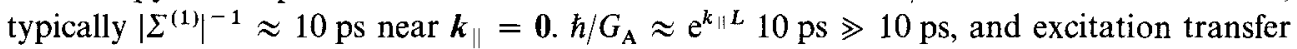
is slow. Consequently, excitons satisfying the IL in MQWs based on GaAs/AlGaAs or other direct-gap semiconductors are likely to dephase more quickly. Moreover, there will be a population of small- $k_{\|}$states whose dynamics are not described by the IL. An alternative for the study of the IL is the use of indirect-gap type-I exciton systems such as InAs/GaAs under proper strain conditions.

(ii) Unlike (i), the LWA can be studied directly in the far field (in $k_{\|}<x$ ), since the exciton-like states here couple directly with the free-space modes of the electromagnetic field. As in (i), the time scale $\hbar / G_{\mathrm{B}} \gg\left|\Sigma^{(1)}\right|^{-1}$. Because $G_{\mathrm{B}}$ does not depend upon $L$ exponentially, the time scale can be fast. For example, for a GaAs/AlGaAs MQW, $x_{\mathrm{ex}}^{-1} \approx 40 \mathrm{~nm}$. If $L=150 \mathrm{~nm}\left(L_{z}=80 \mathrm{~nm}\right.$, say $)$, then $\hbar / G_{\mathrm{B}} \approx 27 \mathrm{ps}$ if $\left|\Sigma^{(1)}\right|^{-1}=10 \mathrm{ps}$. Moreover, we point out the very rapid rise dynamics shown in Fig. 2 b. Thus, long-range coupling is conductive to fast excitation transfer. This is desireable for switching applications, although the population decay is slow. This problem is not expected to be as severe in MQWs composed of just a few QWs where the radiative depletion of the coherent exciton population is on the $10 \mathrm{ps}$ time scale.

Fig. 3 shows proposed excitation and detection schemes for coherent vertical exciton transport. In Fig. 3a, QW B is tuned so its hh resonance lies between the hh and $\mathrm{lh}$ excitons of QWs $A$ and $A^{\prime}$. ( $A^{\prime}$ hh is resonant with $\left.A\right)$. The exciting pulse is then tuned to the hh absorption of $B$, which then transfers excitation to $A^{\prime}$. Similarly, for detection, the hh resonance of $\mathrm{A}$ and $\mathrm{A}^{\prime \prime}$ lies between the $\mathrm{hh}$ and $\mathrm{lh}$ resonances of $\mathrm{C}$. One then time resolves the emission from the hh exciton in $\mathrm{C}$. 

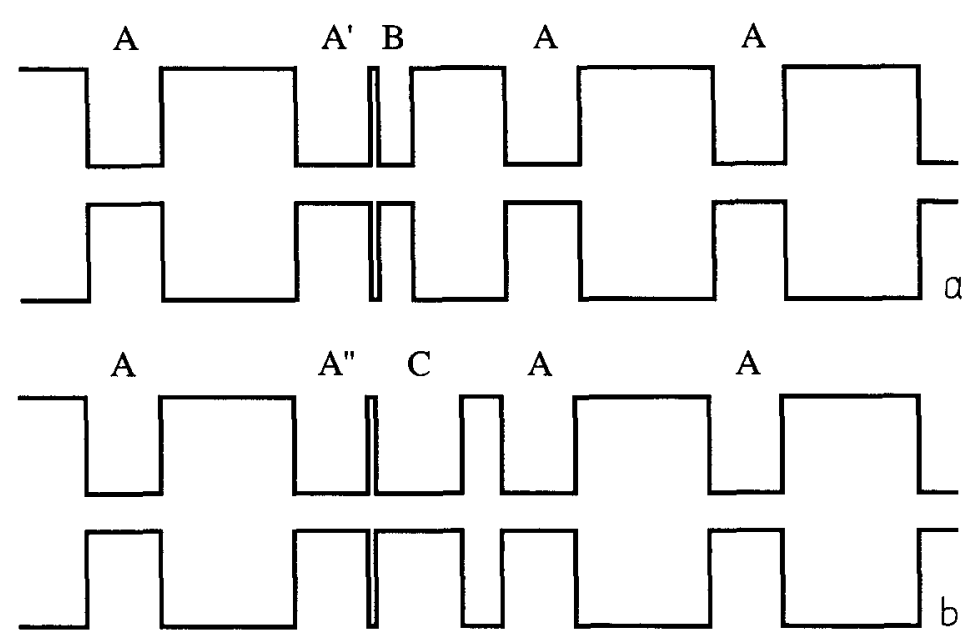

Fig. 3. a) Excitation scheme, b) detection scheme

We conclude this section with the remarks that the infinite-range coupling in planar systems is an essential feature; the neglect of retardation leads to spurious qualitatively different dynamics in the LWL. In finite MQW stacks the dynamics will also contain an oscillatory component due to the reflection of the last QWs. In addition, we expect that the rapid excitation transfer will also be present in the finite stacks, without the long population decay in infinite structures, thus making the phenomena under investigation of interest for applications in optical switching.

\section{Concluding Remarks}

We have outlined two types of polariton effects in MQWs: the disorder-induced origin of excitonic radiative widths in a MQW possessing weak departures from perfect periodicity in the growth direction and coherent vertical transport of excitons in MQWs. The models presented are valid provided other relaxation mechanisms and the non-bosonic character of the exciton may be neglected. To some extent these assumptions hold insofar as the initial transients in low-temperature time-resolved spectroscopic experiments on high-quality structures. In order to obtain a more satisfactory picture, other relaxation processes, such as those associated with exciton-exciton and exciton-free-carrier scattering, with phonon emission and absorption, and various sources of static disorder must be included. Nevertheless, the polariton picture even in its simple form has proven a useful picture in understanding radiative processes in semiconductor quantum structures.

\section{Acknowledgements}

We are grateful to T. B. Norris, J.-K. Rhee, L. G. Gerchikov, D. G. Steel, A. C. Schaefer, and A. Migus for helpful conversations. This work was supported by the National Science Foundation through the Fellows Program at the Center for Ultrafast Optical Science under grant number STC PHY 8920108. 


\section{References}

[1] J. J. Hopfield, Phys. Rev. 112, 1555 (1958).

[2] E. Hanamura, Phys. Rev. B 38, 1228 (1988).

M. Orrit, C. Aslangul, and P. Kottis, Phys. Rev. B 25, 7263 (1972).

S. JORDA, U. RösSler, and D. BRoIDo, Superlattices and Microstructures 12, 85 (1992).

J. K. Jenkins and S. Mukamel, J. chem. Phys. 98, 7046 (1993).

[3] V. M. Agranovich and A. O. DubovskiI, Soviet Phys. - J. exper. theor. Phys. Letters 3, 223 (1966).

[4] L. C. Andreani, F. Tassone, and F. Bassani, Solid State Commun. 77, 641 (1991).

[5] D. S. Citrin, Solid State Commun. 84, 281 (1992); Phys. Rev. B 47, 3832 (1993).

[6] D. S. Citrin, Solid State Commun. 89, 139 (1994); Phys. Rev. B 49, 1943 (1994).

[7] G. D. Mahan, Many-Particle Physics, Plenum Press, New York 1981.

[8] L. C. Andreani and F. Bassani, Phys. Rev. B 41, 7536 (1990).

L. C. Andreani, F. Tassone, and F. Bassani, Solid State Commun. 77, 641 (1991).

[9] J. Feldmann, G. Peter, E. O. Göbel, P. Dawson, K. Moore, C. Foxon, and R. J. Elliot, Phys. Rev. Letters 59, 2337 (1987).

M. Colocci, M. Gurioli, A. Vinattieri, F. Fermi, C. Deparis, J. Massies, and G. Neu, Europhys. Letters 12, 417 (1990).

[10] D. S. Citrin, in: Confined Electrons and Photons: New Physics and Devices, Ed. C. Weisbuch and E. Burstein, Plenum Press, New York, to be published.

[11] D. S. Citrin, Phys. Rev. B 51, to be published.

[12] B. Deveaud, F. Clérot, N. Roy, K. Satzke, B. Sermage, and D. S. Katzer, Phys. Rev. Letters 67, 2355 (1991).

A. Vinattieri, J. Shah, T. C. Damen, D. S. Kim, L. N. Pfeiffer, and L. J. Sham, Solid State Commun. 88, 189 (1993).

[13] R. J. Elliott, J. A. Krumhansl, and P. L. Leath, Rev. mod. Phys. 46, 465 (1974); and references therein.

[14] J. KnOester, Phys. Rev. Letters 68, 654 (1992).

[15] D. S. Citrin, Chem. Phys. Letters, to be published.

[16] B. Deveaud, A. Chomette, B. Lambert, and A. Regreny, Solid State Commun. 57, 885 (1986); B. Devenud, J. Shah, T. C. Damen, B. Lambert, and A. Regreny, Phys. Rev. Letters 58, 2582 (1987).

A. Nakamura, N. Kawamoto, K. Fujiwara, N. Tsukada, and T. Nakayama, Solid State Commun. 71, 553 (1989).

[17] T. FöRSTER, Z. Naturf. 4a, 321 (1949).

[18] I. Yamazaki, N. Tamai, T. Yamazaki, M. Mimura, A. Murakami, and Y. Fujtra, in: Primary Processes in Photobiology, Ed. T. KobaYashi, Springer-Verlag, Berlin 1987.

[19] M. Hauser, U. K. A. Klein, and U. Gösle, Z. phys. Chem. N.F. 101, S255 (1976).

A. Blumen and G. Zumofen, J. chem. Phys. 75, 892 (1981).

G. Zumofen and A. Blumen, J. chem. Phys. 76, 3713 (1982).

J. BAUMANN and M. D. FAYER, J. chem. Phys. 85, 4087 (1986).

[20] L. G. Gerchikov and A. V. Subashiev, Solid State Commun. 90, 57 (1994).

[21] A. Tomita, J. Shah, D.-S. Kim, T. C. Damen, J. M. Kuo, S. SchmitT-Rink, P. Thomas, and R. S. KNox, in: Quantum Electronics and Laser Science Conference, 1992, OSA Technical Digest Ser., Vol. 13, Optical Society of America, Washington, DC, 1992 (pp. 116 to 118).

[22] L. C. ANDrEANI, phys. stat. sol. (b) 188, 29 (1995). 